\title{
Food allergy (Clinical case)
}

\author{
Alergia alimentar (Caso clínico)
}

\section{Ariana Saraiva ${ }^{1}$, António Raposo ${ }^{2}$}

${ }^{1}$ Pharmacy Faculty, University of Coimbra, Azinhaga de Santa Comba, 3000-548 Coimbra, Portugal. ${ }^{2}$ CBIOS (Reserch Center for Biosciences and Health Technologies), Universidade Lusófona de Humanidades e Tecnologias, Campo Grande 376, 1749-024 Lisboa, Portugal.

Email: antonio.raposo@ulusofona.pt

A 27-year-old student woman has symptoms of allergic reaction when she eats eggs or peanuts. It manifests with a skin irritation. She has inclusive severe allergic reaction, feeling weakness or difficulty breathing. General practitioner medicated her with Zyrtec. She was sent to the Nutrition Consultation to help her to choose the right foods, avoiding allergic ingredients.

\section{Anthropometric evaluation}

Weight: $53 \mathrm{~kg}$

Height: $164 \mathrm{~cm}$

BMI: $19.9 \mathrm{~kg} / \mathrm{m}^{2}$

Waist circumference: $90 \mathrm{~cm}$

Assessment of body composition (TANITA BC-545N)

Body fat: $21.6 \%$

Total body water: $58.1 \%$

Analytical Parameters:

\section{Hematology}

Leukocytes: 6.7 x 10\%/L (4.0-10.0)

Erythrocytes: $4.53 \times 10^{12} / \mathrm{L}(3.90-5.20)$

Hemoglobin: 13.5 g/dL (12.0-16.0)

Hematocrit: $39.2 \%$ (34.7-46.0)

Glycated hemoglobin (A1C): 5\% $(<5.7)$

\section{Biochemistry}

Glucose: $66 \mathrm{mg} / \mathrm{dL}(50-100)$

Urea: $27 \mathrm{mg} / \mathrm{dL}(<50)$

Creatinine: $0.68 \mathrm{mg} / \mathrm{dL}(<1.2)$

Uric acid: $2.5 \mathrm{mg} / \mathrm{dL}(2.4-6.0)$

Total cholesterol: $137 \mathrm{mg} / \mathrm{L}(<200)$
Received /Recebido: 15/04/2019 Accepted / Aceite: 01/10/2019

Electronic Edition: www.alies.pt

Uma estudante de 27 anos apresenta sintomas de reacção alérgica ao ingerir ovos ou amendoins. Esta alergia manifesta-se através de uma irritação da pele. Ela tem reacção alérgica grave inclusive, sentindo fraqueza ou dificuldade em respirar. O médico de clínica geral medicou-a com Zyrtec e reencaminhou-a para a Consulta de Nutrição para poder escolher os alimentos certos, evitando ingredientes alérgicos.

Avaliação antropométrica

Peso: $53 \mathrm{~kg}$

Altura: $164 \mathrm{~cm}$

IMC: $19,9 \mathrm{~kg} / \mathrm{m}^{2}$

Circunferência da cintura: $69 \mathrm{~cm}$

Avaliação da composição corporal (TANITA BC-545N)

Gordura corporal: $21,6 \%$

Água corporal total: $58,1 \%$

Parâmetros analíticos:

Hematologia

Leucócitos: 6,7 x109 / L (4,0-10,0)

Eritrócitos: 4,53 x 1012 / L (3,90-5,20)

Hemoglobina: 13,5 g / dL (12,0-16,0)

Hematócrito: $39,2 \%(34,7-46,0)$

Hemoglobina glicada (A1C): $5 \%(<5,7)$

\section{Bioquímica}

Glicose: 66 mg / dL (50-100)

Uréia: $27 \mathrm{mg} / \mathrm{dL}(<50)$

Creatinina: $0,68 \mathrm{mg} / \mathrm{dL}(<1,2)$

Ácido úrico: 2,5 mg / dL (2.4-6.0)

Colesterol total: $137 \mathrm{mg} / \mathrm{L}(<200)$ 
HDL cholesterol: $56 \mathrm{mg} / \mathrm{L}(>59)$

LDL cholesterol: $78 \mathrm{mg} / \mathrm{L} /<130)$

Triglycerides: $56 \mathrm{mg} / \mathrm{L}(<150)$

Alanine Aminotransferase: $8 \mathrm{U} / \mathrm{L}(<45)$

Aspartate Aminotransferase: $17 \mathrm{U} / \mathrm{L}(<32)$

Gama Glutamiltransferase: 9 U/L $(<42)$

Iron: $139 \mu \mathrm{g} / \mathrm{dL}(60-170)$

\section{Hormonology}

Thyroid Function:

Free T4: $1.2 \mathrm{ng} / \mathrm{Dl}(0.7-1.8)$

TSH: $2.8 \mathrm{mUI} / \mathrm{L}(0.4-4.2)$

Anemia

Ferritin: $16.6 \mu \mathrm{g} / \mathrm{L}(50.0-200.0)$

Folic Acid: $2.9 \mu \mathrm{g} / \mathrm{L}(>4.5)$

Vitamin B12: $432 \mu \mathrm{g} / \mathrm{L}(>200)$

Clinical evaluation:

Personal background:

Asthma

Allergic rhinitis

Medication:

Zyrtec

Intestinal transit: regular

Eating habits:

Wake up at 9:00 a.m.

Breakfast: cereals with chocolate

During morning: 1 apple

Lunch: 1:00 p.m.

Dish: half a plate with noodles or rice and the other with meat $(100 \mathrm{~g})+\operatorname{salad}(30 \mathrm{~g})$

Drink water $(200 \mathrm{ml})$

During afternoon:

milk with chocolate $(220 \mathrm{ml})+$ one bread with one slice of ham

Yogurt with strawberry

Dinner: 8:00 p.m.

One plate of soup

Between dinner and time to sleep: one apple

Lie down: 12 p.m.

Water consumed: about 1 L per day

Environment, behavior and social:

She does exercises about 5 days per week.

She has the habit to snack while studying and likes to
Colesterol HDL: $56 \mathrm{mg} / \mathrm{L}(>59)$

Colesterol LDL: $78 \mathrm{mg} / \mathrm{L} /<130)$

Triglicerídeos: $56 \mathrm{mg} / \mathrm{L}(<150)$

Alanina aminotransferase: $8 \mathrm{U} / \mathrm{L}(<45)$

Aspartato aminotransferase: $17 \mathrm{U} / \mathrm{L}(<32)$

Gama Glutamiltransferase: 9 U / L $(<42)$

Ferro: $139 \mu \mathrm{g} / \mathrm{dL}$ (60-170)

\section{Hormonologia}

Função tiroideia:

T4 livre: $1,2 \mathrm{ng} / \mathrm{Dl}(0,7-1,8)$

TSH: 2,8 mUI / L $(0,4-4,2)$

Anemia

Ferritina: 16,6 $\mu \mathrm{g} / \mathrm{L}(50,0-200,0)$

Ácido fólico: $2,9 \mu \mathrm{g} / \mathrm{L}(>4,5)$

Vitamina B12: $432 \mu \mathrm{g} / \mathrm{L}(>200)$

Avaliação clínica:

Antecedentes pessoais:

Asma

Rinite alérgica

Medicação:

Zyrtec

Trânsito intestinal: regular

Hábitos alimentares:

Acorda às 9:00

Pequeno-almoço: cereais com chocolate

Durante a manhã: 1 maçã

Almoço: 13:00

Prato: meio prato com massa ou arroz e outra metade com carne (100 g) + salada (30 g)

Bebe água $(200 \mathrm{ml})$

Durante a tarde:

leite com chocolate $(220 \mathrm{ml})+$ um pão com uma fatia de presunto

Iogurte de morango

Jantar: 20:00

Um prato de sopa

Entre o jantar e a hora de dormir: uma maçã

Deita-se às 24:00

Água consumida: cerca de $1 \mathrm{~L}$ por dia

Ambiente, comportamento e social:

A estudante faz exercícios cerca de 5 dias por semana. Ela tem o hábito de fazer um lanche enquanto estuda 
eat in fast food restaurants.

She does not like to eat potatoes and does not eat much fish nor vegetables.

\section{Questions:}

1 - What is an allergic reaction?

2 - Is the allergic reaction to food dangerous?

3 - The food to which she is allergic compromised a balanced diet?

4 - What foods she has to avoid?

\section{Answers:}

1 - An allergic reaction is an adverse reaction produced by immune system because of the exposition to an allergen. It can be IgE-mediated and no IgE-mediated or the two responses. When IgE antibody is produced there is development of symptoms. $(1,2)$

2 - The allergic reaction to food can be very dangerous because of allergic anaphylaxis. This is a severe response in susceptible individuals that can lead to death or permanent damage. It is essential to avoid the exposure of allergenic factors that can lead to exacerbation. (3-5)

3 - When someone has an allergic reaction to a complete food such eggs, composed of quality proteins, vitamins and minerals, it is necessary to replace with an increase of vegetables, cereals, fruits and red meat. (6) 4 - She has to avoid all foods with the allergenic factors: peanuts and eggs. She cannot eat cakes with eggs, only vegan eggs, nor can she eat ice cream and noodles that have egg in their composition. She needs to be careful with sauces normally used in sandwiches, such as mayonnaise. It is very important that she ask before buying every product with potential allergic ingredients. (7) Some individuals that have allergic reactions to eggs are also sensitive to poultry meat, so its avoidance is recommended. She must read the labels of food to guarantee the absence of eggs due to their extensive utilization. $(1,8)$ (na maioria das vezes come bolachas) e gosta de comer em restaurantes de fast food.

Ela não gosta de comer batatas e não come muito peixe nem legumes.

Questões:

1. O que é uma reação alérgica?

2. A reacção alérgica à comida é perigosa?

3. A comida que a estudante é alérgica compromete uma dieta equilibrada?

4. Quais alimentos que ela tem de evitar?

Respostas:

1. Uma reacção alérgica é uma reacção adversa produzida pelo sistema imunológico devido à exposição a um alergénio. Pode ser mediada por IgE e não mediada por IgE ou poderá ter duas respostas. Quando o anticorpo IgE é produzido, há desenvolvimento de sintomas. $(1,2)$

2. A reacção alérgica aos alimentos pode ser muito perigosa devido à reacção de anafilaxia. Esta é uma resposta severa em indivíduos susceptíveis que pode levar à morte ou a danos permanentes. É essencial evitar a exposição de factores alérgicos que podem levar à exacerbação. (3-5)

3. Quando alguém tem reacção alérgica a um alimento completo como os ovos, compostos por proteínas, vitaminas e minerais de qualidade, é necessário repor estes nutrientes aumentando o consumo de vegetais, cereais, frutas e carne vermelha.(6)

4. Ela tem de evitar todos os alimentos com factores alérgicos: amendoins e ovos. Não pode comer bolos com ovos, somente pode ingerir os bolos veganos, não pode consumir gelados e massa com ovo na sua composição. Tem de ter cuidado com os molhos normalmente usados para rechear as sandes, como a maionese. É muito importante questionar antes de comprar todos os produtos com potenciais ingredientes que lhe provocam alergia.(7) Algumas pessoas que têm reacções alérgicas aos ovos também são sensíveis à carne de aves, por isso é recomendável evitá-la. A estudante precisa de ler os rótulos dos alimentos para garantir a ausência de ovos devido à sua ampla utilização. $(1,8)$ 


\section{References/Referências}

1. Carrapatoso, I., Prates, S., \& Costa. C. (2017). Alergia Alimentar: Conceitos, Conselhos e Precauções. Brasil, Thermo Fischer, 1 Ed., p.103-111.

2. Valenta, R., Hochwallner, H., Linhart, B., \& Pahr, S. (2015). Food allergies: the basics. Gastroenterology, 148(6), 1120-1131.

3. Raposo, A., Pérez, E., Tinoco de Faria, C., \& Carrascosa, C. (2017). Allergen Management as a Key Issue in Food Safety. In Food Safety and Protection (pp. 195-241). CRC Press.

4. Ferreira, H., Ferreira, C., Silva, A., Costa, A., \& Pedrosa, C. (2015). Anafilaxia e alergia alimentar: O resultado de uma intervenção na comunidade. Nascer e Crescer, 24(3), 103-107.

5. Sarinho, E., \& Lins, M. D. G. M. (2017). Formas graves de alergia alimentar. Jornal de Pediatria, 93(1), 53-59.

6. Worm, M., Eckermann, O., Dölle, S., Aberer, W., Beyer, K., Hawranek, T., ... Ruëff, F. (2014). Triggers and treatment of anaphylaxis: an analysis of 4000 cases from Germany, Austria and Switzerland. Deutsches Ärzteblatt International, 111(21), 367.

7. Turner, P. J., Baumert, J. L., Beyer, K., Boyle, R. J., Chan, C. H., Clark, A. T., ... \& Grabenhenrich, L. (2016). Can we identify patients at risk of lifethreatening allergic reactions to food?. Allergy, 71(9), 1241-1255.

8. Turnbull, J. L., Adams, H. N., \& Gorard, D. A. (2015). The diagnosis and management of food allergy and food intolerances. Alimentary Pharmacology \& Therapeutics, 41(1), 3-25. 